\title{
Impact of Pinch Strength Training along with Postural Education on Upper Extremity Functional Performance among Dentist
}

\author{
Simulia Dhinju B ${ }^{1}$, Paulraj $\mathbf{M}^{2}$, Manjula Devi $\mathrm{K}^{3}$ \\ ${ }^{1}$ MPT (Neuro), Assistant Professor, College of Physiotherapy, Sri Venkateshwaraa College of Paramedical \\ Sciences, Puducherry, India \& Sub Coordinator, Indian Association of Physiotherapy Women Cell, India. \\ ${ }^{2} \mathrm{PhD}$ Scholar (NIT) \& Associate Professor, College of Physiotherapy, Sri Venkateshwaraa College of \\ Paramedical Sciences, Puducherry, India. Pin code: 605102 \\ ${ }^{3}$ BPT Intern, College of Physiotherapy, Sri Venkateshwaraa College of Paramedical Sciences, Puducherry, \\ India. Pin code: 605102
}

Corresponding Author: Simulia Dhinju B

\begin{abstract}
Background: Dentists are required to maintain a high level of precision during manual scaling, polishing and Flossing. As a result of sustained griping activity finger and wrist pain are more common among Dentists. Therefore the excess pinch force, leads to musculoskeletal disorders which in turn affects the functional ability.
\end{abstract}

Study design: An Experimental study.

Materials \& Methods: The sums of 30 Dental professionals were selected under inclusion criteria into this study, ages between 20 to 35 years with decrease in pinch strength and functional abilities. Selected subjects were randomly allocated into two groups such as experimental group (Pinch strength training and conventional exercise) and control group (Conventional Exercises - Postural corrective exercises, active range of motion exercises, shoulder bracing and shoulder shrugging exercise, Ergonomics advices) were given. The outcome tools used are Modified Sphygmomanometer test and Disabilities of the Arm, Shoulder and Hand Questionnaires (DASH). The Statistical analysis of pre \&post test values were analyzed using paired \& unpaired ' $t$ ' test.

Result: The result of this study shows that Group A using Pinch strength along with Postural Education were found to be effective over the control group, where only the postural education were done $(\mathrm{p}<0.0001)$.

Conclusion: Therefore this study concludes that Pinch strength training along with Postural education was proven to be significant in improving Pinch strength and Functional performance among Dentist.

Keywords: DASH Questionnaires, Functional disabilities, Modified Sphygmomanometer test and Pinch strength training.

\section{INTRODUCTION}

Dental practice is a high risk profession and large number of dentists are at risk of job related musculoskeletal disorder. $^{[1,2]}$ There are high level prevalence of neck $(75.74 \%)$, wrist/hand $(73.13 \%)$, lower back $(72.01 \%)$, Shoulder $(69.47 \%)$, hip (29.85\%), upper back (18.65\%), ankle
$(12.31 \%)$ and elbow $(7.4 \%)$ in 646 dentist. ${ }^{[2,3]}$

US department of labor defines work related musculoskeletal disorder (WMSD) as injuries of the tendon, nerves, muscles, spinal disc and joint cartilage associated with risk factors in the work place. ${ }^{[4]}$

Dentists are required high level precision work during dental prophylaxis, 
especially manual scaling, involving high level forces and precision to remove hard calculus from small area of the tooth's. ${ }^{[5]}$

Dental professional require small precise movements, especially they use their thumb, index and middle finger in gripping. Highly repetitive work leads to tendon disorder in hand. ${ }^{[6,7]}$ The cause for repetitive motion disorder is of unnatural motions and incorrect posture. That causes pain, loss of strength, numbness, less flexibility. They need forceful pinching or gripping, sustained non neutral wrist position and use of vibrating tools e.g.: Root planning and Scaling resulting in Wrist or hand pain are more due to the sustained grips. If it is untreated it causes permanent damage to muscle, tendons, nerves and ligaments.

There are three types of pinches as follows ${ }^{8}$ Pad to pad type, Tip to tip type and lateral pinch. All this types of pinches are produced by combined action of intrinsic and extrinsic hand muscles. ${ }^{[8,9]}$ The overall function of the intrinsic hand muscles is considered to be fine motor control, while the extrinsic hand muscles function is gross motion performance and major hand forces production. ${ }^{[10]}$

The proper technique of holding the instrument is modified pen grasp. It is grasping the instrument between the thumb and radial aspect of the index and middle fingers in a tripod fashion. Pinch strength was affected with increasing disability and because of repetitive work by hand and wrist. $^{[11,12]}$

Pinch strength was usually measured by pinch gauge. There is an availability of Modified sphygmomanometer test (MST) which is another method that can be applied in clinical settings for the assessment of muscle strength including pinch strength. In this procedure pad to pad pinch, tip to tip pinch and lateral pinch are measured. ${ }^{[13,14]}$ Presence of disability followed by neck \& hand is measured by DASH 30- items disability scale. ${ }^{[15]}$ and then the application of hand muscles strengthening exercises are essential. ${ }^{[16,17]}$ Recent studies states that the strength training along with appropriate posture could improve the muscle strength $\&$ its efficiency ${ }^{[18-22]}$

Therefore, this study focuses on improving the Pinch strength and functional performance among Dentist by pinch strength training along with postural advice.

\section{MATERIALS AND METHODOLOGY}

It is experimental study which includes the dentist population. The interventions were planned for about 4 weeks, 5 sessions each week. Totally 30 Dentist ware included.

Selection Criteria: Subjects ages between 20-30 years, both male \& female Dentists (with minimum I year of experience) having moderate to severe disability assessed using DASH Questionnaire Subjects and Modified sphygmomanometer test were included. Disabling neurological conditions such as cervical radiculopathy, cervical myelopathy or any other recent trauma to neck and upper limb were excluded.

\section{Outcome measures: \\ Modified sphygmomanometer test. ${ }^{[13,14]}$}

Pinch strength was measured by Modified sphygmomanometer test (MST). To remove the wrinkles in BP cuff it should be inflated to $100 \mathrm{~mm} \mathrm{hg}$ and it should be maintain at baseline pressure of $20 \mathrm{~mm} \mathrm{Hg}$. The valve should be closed tightly to prevent air leakage. Each participant was advised to seated, elbow flexed to $90^{\circ}-100^{\circ}$, forearm in pronation and then wrist extended to $0-30^{\circ}$ to hold the cuff. And then 3 trials were assessed with 30 seconds rest and mean values were recorded.

\section{DASH (Disabilities of the Arm, Shoulder and Hand questionnaire $)^{[15]}$}

It is a self administered questionnaire, as a measure of upper extremity disability and symptoms of several musculoskeletal disorders. DASH consists of a 30 items, scored from 0 (no disability) to 100 . The score for each participant was calculated as: DASH SCORE $=[($ sum of $\mathrm{n}$ responses / $\mathrm{n})-1] \mathrm{x}$ 25 , where $\mathrm{n}$ is the number of completed 
responses). The questionnaire may not be calculated if there is more than 3 items missing.

Study protocol: 30 Dentists was selected as per the inclusion and exclusion criteria.

\section{Procedure:}

\section{Group -A (Pinch strength training) ${ }^{[8]}$}

The subject has to be seated in a comfortable position and clearly explained about the procedure. Pinch strength of the dominant hand (pad to pad, tip to tip, lateral pinch) where measured and hand muscle strengthening exercises were given to them. The strengthening exercises are ball squeezing, elastic band exercise, closed fist, fingers apart, closed fingers, opposition of thumb with digits, knuckle bend exercise. Each exercise for 30 repetitions per day, weekly 5 session for 4 week.

\section{Conventional exercises ${ }^{[23,24,25]}$}

Shrugging exercise: Straight standing with relaxed shoulder, The subject is asked to keep the arms at the side with the elbow in a fully extended position. The subject is asked to raise the shoulder as high as possible and ask them not to roll the shoulder backwards and not to make arch of their back. The subject is asked to lower the shoulder slowly. 10 repetitions with 5 seconds hold should be done daily.

Bracing exercise: Straight standing with relaxed shoulder. The subject is asked to abduct both the shoulder with elbow in semi flexed position by placing the two hands one above the other near the chest level (protraction of the scapula). Then the subject is asked to slowly move the hand out by retracting the scapula by squeezing the shoulder blades. 10 repetitions with 5 second hold should be done daily.

AROM exercises for upper extremities: Each movement done with 10 repetition for about 30 times. $10 \mathrm{sec}$ rest per 10 repetition in a comfortable position.

\section{Postural awareness education ${ }^{[22]}$}

To maintain an erect posture: By positioning the patient close to the chair, to minimize forward bending. Place the feet flat to promote a pelvis in neutral position and natural curvatures of back.

Minimize excessive wrist movements: To keep the wrist in a neutral position (palms facing each other, shoulder are width apart and with wrist straight).

Avoid excessive finger movements: The excessive forces on fingers are needed to hold the instruments. So retraining the shoulders and arms in proper position hand, to avoid forceful movements with fingers.

\section{Ergonomic advice: (Fig. 1 \& 2)}

Awareness of the postures to reduce the stress among the Dental worker. By practicing the correct postures, that can improve the quality of Dental practice.

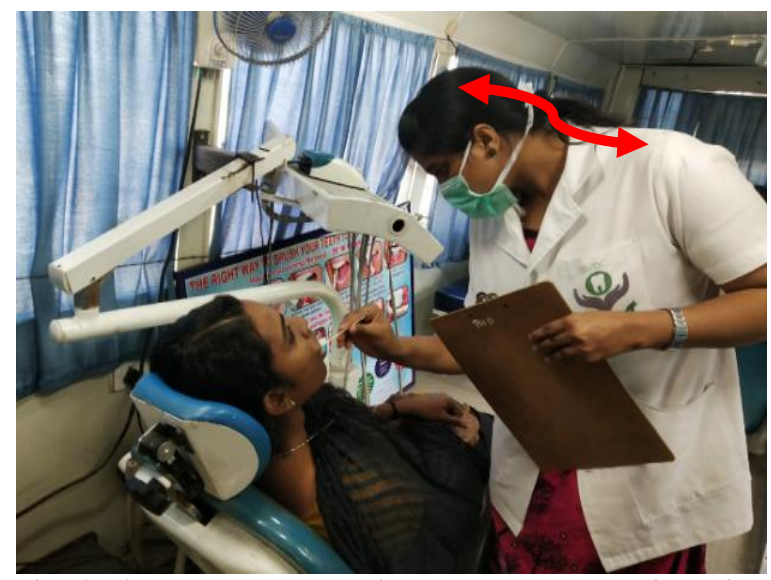

Fig. 1- Arrow denotes the Abnormal Posture while doing Dental procedure before training with Pinch strength training \& Postural Education

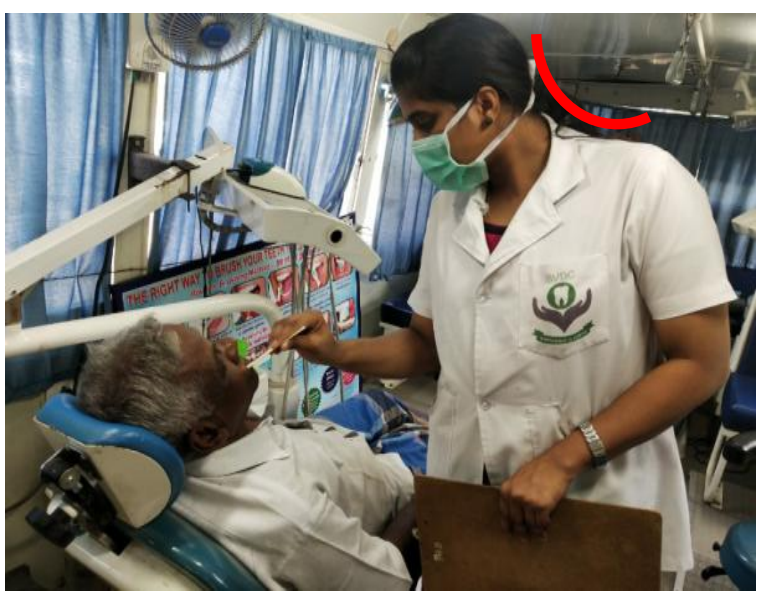

Fig. 2 - Ideal Postural while doing the Dental Procedure post training with Pinch strength training \& with Postural Education 


\section{Data analysis}

Pre and post interventional differences within the two groups were analyzed using paired ' $\mathrm{t}$ ' test and between the two groups were analyzed using unpaired ' $t$ ' test for each of the outcome measures mentioned in the below tables (Table 1). Statistical significance was set at $\mathrm{p}<0.0001$.

Table 1: Results of Statistical Analysis using unpaired't' test (Between the group)

\begin{tabular}{|c|c|c|c|c|}
\hline GROUP & Mean & SD & t-value & p-value \\
\hline \multicolumn{5}{|c|}{ Pad-pad pinch } \\
\hline Group-A & 74.33 & 6.78 & 2.6536 & 0.01 \\
\hline Group-B & 66.33 & 9.51 & & \\
\hline \multicolumn{5}{|c|}{ Tip-tip pinch } \\
\hline Group-A & 77 & 5.61 & 2.6827 & 0.01 \\
\hline Group-B & 68.5 & 10.86 & & \\
\hline \multicolumn{5}{|c|}{ Lateral pinch } \\
\hline Group-A & 73.33 & 6.17 & 2.7929 & 0.001 \\
\hline Group-B & 65.93 & 8.02 & & \\
\hline \multicolumn{5}{|l|}{ DASH } \\
\hline Group-A & 17.27 & 5.97 & 2.1425 & 0.01 \\
\hline Group-B & 22.87 & 8.18 & & \\
\hline
\end{tabular}

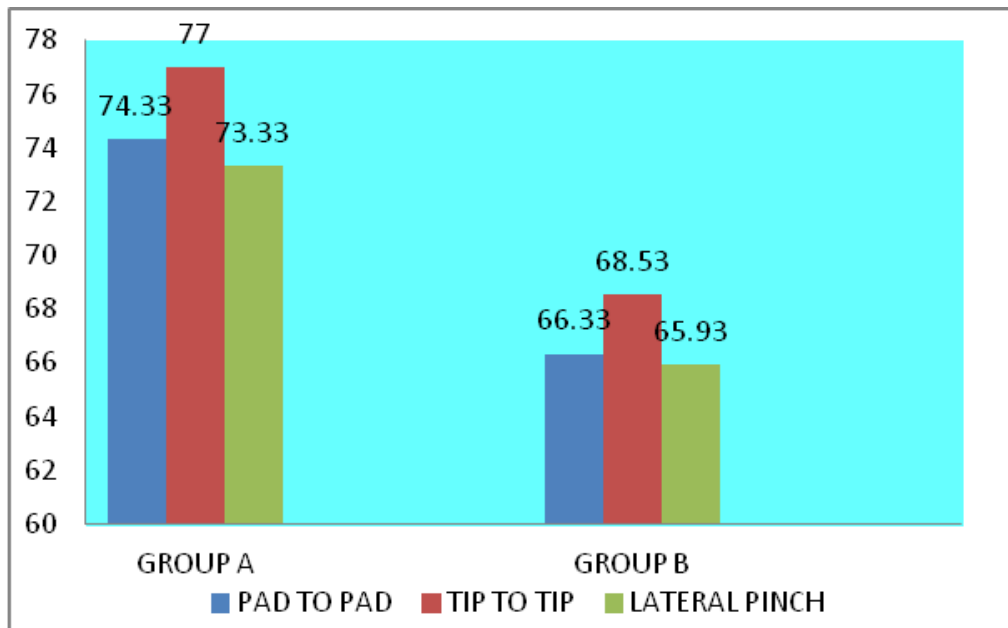

Fig. 3 - Between the group analysis of pad to pad pinch, tip to tip pinch, lateral pinch.

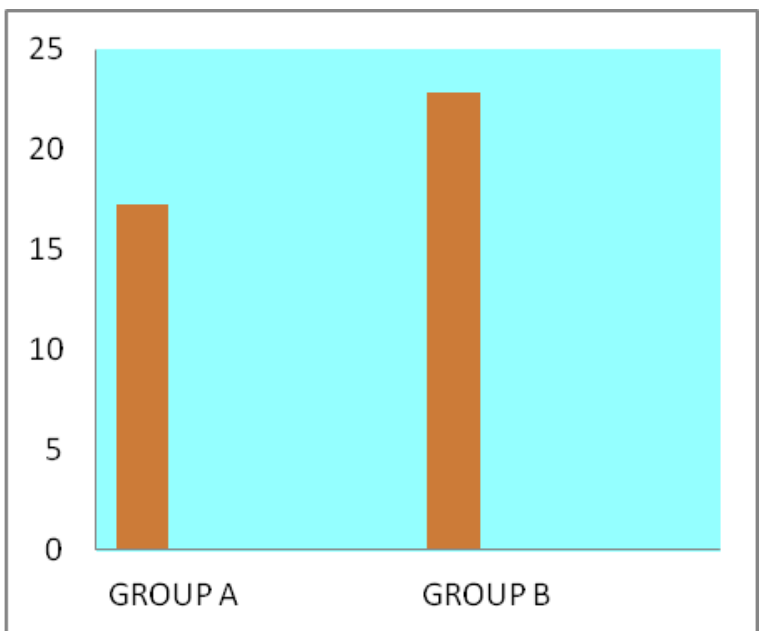

Fig 4 - Between the group analysis of functional disability (Group A\& B) using DASH Questionnaire.

\section{RESULTS}

The statistical analysis done using unpaired ' $\mathrm{t}$ ' test with the values of experimental and control group shows significance of $(p<0.0001)$. Between the group analysis of the post value shows experimental group is significant than the control group. After the statistical analysis, it shows that there is improve in pinch strength and functional performance in experimental group A (pinch strength training and conventional exercise.) than control group B (conventional exercise alone) which shows that the experimental group is significant than the control group (Fig $3 \& 4$ ).

\section{DISCUSSION}

Most of the Dentist in their life time is getting affected due to the habitual life style. Therefore in this study 30 subjects were selected on the basis of selection criteria and randomly divided into two groups, 15 each. Group-A experimental group ( $\mathrm{n}=15$; pinch strength training) and Group-B control group ( $n=15$; Ergonomics advices). The outcome measures used to assess the pinch strength and function performance were modified sphygmomanometer test and DASH (Disability of arm, shoulder, hand questionnaire). Both the groups showed improvement in the pinch strength and function performance followed by the 5 
treatment sessions. According to some studies hand and wrist related musculoskeletal disorders are more common among dentist due to their specific procedure that are performed for longer time. In the experimental group pinch strength training was administered. This technique including ball squeezing, elastic band exercise, closed fingers, closed fist, closed apart, opposition of thumb with digits, knuckle bend exercises, finger stretching exercise. ${ }^{[8]}$

Effects of strength training: It is a physical exercise, which gives resistance to muscles that builds the anaerobic endurance, strength and size of skeletal muscle. The benefits of strengthening exercises are to increase the energy levels of muscles and to decrease muscle fatigue level. ${ }^{[18,19]}$

Strength training is considered to be progressive resistance exercise but intervention that repetitive effortful muscle contractions can result in increased motor unit activity, thereby potentially increasing strength. ${ }^{[20]}$ It is known that muscle strength can be improved by strength training program. ${ }^{[21]}$ Awareness of the ideal postures among Dentist is to reduce the stress on their body and by practicing with correct postures in working can improve the quality of Dental practice. ${ }^{[22]}$

Strength training improves the muscle force by improving acquisition, frequency and synchronization of motor units, thereby enhances Muscle strength. ${ }^{[18]}$ Nikolaus Johannes Wachter et al.,(2017) concluded that the measurement of tip pinch, tripod pinch and key pinch can improve the follow up in hand rehabilitation. ${ }^{[19]}$ Jose Manuel PerezMarmol et al.,(2017) stated that exercise program for fine motor skills was effective to improve manual dexterity and also the active and passive range of motion. That is specific interventions of the hand are needed to prevent a worsening in range of finger motion in hand osteoarthritis. ${ }^{[17]}$ Therefore Strengthening exercise for hand muscles help to improve the pinch strength among Dentist. It may be due to the physiological and therapeutic effects of exercises such as increased requirements for oxygen, removal of metabolites waste and carbon dioxide. ${ }^{[21]}$

From the above statement, pinch strength training seems to be more effective in improving the pinch strength and functional performance among Dentist. Along with the pinch strength training the experimental group subject received conventional exercises. The effect of the conventional exercise for both experimental and control groups could have strengthened the weak musculature of the Upper extremity, reduces the muscle spasm with given appropriate Postural correction exercises and Ergonomics advices. By practicing with correct postures the working capacity and productivity of Dental professionals can be enhanced and thus can improve the quality of dental practice.

\section{CONCLUSION}

Therefore this concluded that the Pinch strength training along with postural education is effective in improving upper extremity functional performance among Dentist. Hence the Null Hypothesis is rejected. Dentists are advised to use these exercises on regular basis to obtain long term effects.

\section{Limitations \& recommendations:}

Limitations of this study were condensed sample size and treatment duration was really short (4 week). Subjects were selected from same Dental College. Pinch strength was not assessed in Non-dominant hand. The recommendation of this study is other outcome measures like pinch gauge could be used and Study population can be selected from various professionals who uses hand dexterities for a longer time; other conditions like upper limb never injury, carpel tunnel syndrome etc., can also be considered into such Studies. The impact of Pinch strength training could be compared between the Dominant \& Non dominant Hands and other beneficial exercises like EMG Bio feedback could be used in the training of intrinsic muscles of hand. 


\section{ACKNOWLEDGEMENTS}

Heartfelt thankfulness to Prof. Dr. A. Pahinian MPT, MBA, PhD., our Principal college of physiotherapy and colleagues for the extended support, to all my participants of Sri Venkateshwaraa Dental College for their persistent participation.

\section{Conflict of interest}

The authors declare that they have no conflict of interest regarding the publication.

\section{Funding Source}

The current study was selfsupported.

\section{Ethical Approval: Approved}

\section{REFERENCES}

1. Kumar VK, Kumar SP, Baliga M Retal., Prevalence of work-related musculoskeletal complaints among dentists in India: A national cross-sectional survey. Indian $\mathbf{J}$ Dent Res 2013; 24:428-38.

2. Anand Heggannavar, ShivaniKurode, Santosh Metgud, Upper Extremity and Neck Disability In Dentist with Concurrent Changes In Pinch Strength: an Observational Study. Asian Journal of Medical and Health Research 2017;2(3), ISSN: 2455-8664.

3. ERIC E.J. RAVEN, DANIEL HAVERKAMP et al., "Construct Validity and Reliability of the Disability of Arm, Shoulder and Hand Questionnaire for Upper Extremity Complaints in Rheumatoid Arthritis". The Journal of Rheumatology 2008; 35:12; doi:10.3899/jrheum.080067.

4. Bureau OfLabor Statistics News, United States Department of Labor. 2004

5. Valachi.B, Valachi. K et al., Mechanisms leading to musculoskeletal disorders in Dentistry, The Journal of The American Dental Association, 2003, vol. 134, no.10, pp.1344-1350

6. Werle S, Goldhahn J, Drerup S, et al. Ageand gender-specific normative data of grip and pinch strength in a healthy adult Swiss population. Journal of Hand Surg 2009 Feb; 34(1):76-84.
7. SyedaUzma Abbas et al., Pinch Force and Work related Musculoskeletal Disorders in Dental Professionals. University of Connecticut Health centre Graduate School. 2004;

8. D. Malarvizhi, A.Abinayaetal., "Effectiveness of Hand Muscle Strenthening Exercises on Dominant Hand Pinch Strength in Smartphone Addicted Collegiate". Indian Journal of Public Health Research \& Development, March2019, Vol.10, No.3.

9. Levangie, P. K., \&Norkin, C.et al., Joint structure and function: A comprehensive analysis. Philadelphia, PA: F.A. Davis Co (2005).

10. Dr. P. Sathya,Dr.VasanthiKadhiravan and PrarthanaPrakashPoojary. Effect of resisted exercises versus free weight exercises for the improvement of grip strength of cricket players. International Journal of Advanced Research,Vol- 4, Issue 7, (1193-1198).

11. Atwood-Sanders, M., \&Michalak-Turcotte, C, Strategies to reduce work-related musculoskeletal disorders in dental hygienists: two case studies. Journal of Hand Therapy, 15(4), 363-374, (2002).

12. Akesson.I, G. Lundborg, V. Horstmann, S. Skerfving, Neuropathy in female Dental personnel exposed to high frequency vibrations, Occupational and environmental medicine, 1995, vol.52, no.2, pp. 116-123.

13. Lucas A. C. Souza, Julia C. Martins, et al., Assessment of muscular strength with the modified sphygmomanometer test: what is the best method and source of outcome values, Journal Of Physical Therapy. 2014 Mar-Apr; 18(2):191-200.

14. Julia Caetano Martins et al. The reliability and validity of the modified sphygmomanometer test (MST) for assessment of strength of upper limb muscle after stroke. J Rehab Med. 2015; 47: 697705.

15. Nelson Fong Soo Hoo, J Hand Surg. Et al., Comparative Study, Evaluation of the Construct Validity of the DASH Questionnaire by Correlation of the SF-36, 2002 May.

16. Bakar et al., Musculoskeletal disorders among dentists and dental students in West Sumatera, Indonesia. Journal of Dentomaxillofacial Science August 2019, Volume 4 Number 2: 92-95. 
17. Perez-Marmol, Jose Manuel et al. Effectiveness of affine motor skills rehabilitation program on upper limb disability, manual Dexterity, pinch strength, range of fingers motion, performance in activities of daily living, functional independency, and general self-efficacy in hand osteoarthritis: A randomized clinical trial Journal of Hand Therapy, Volume30, Issue 3, 262-273

18. Mayer F, Scharhag-Rosenberger F, Carlsohn A, et al.:The intensity and effects of strength training in the elderly. DtschArzteblInt 2011; 108(21):35964.DOI: $10.3238 /$ arztebl.2011.0359

19. Nikolaus Johannes Wachter,MartinMentzel, Gert D. Krischak,Joachim Quantification of hand function by power grip and pinch strength force measurements in ulnar nerve lesion simulated by ulnar nerve block. Journal of hand therapy : official Journal Of The American Society Of Hand Therapists, ISSN:1545-004X Publication Year:2017

20. Sannam Khanetal., Prevalence of work related musculo-skeletal disorders (MSD) among dentists. International Journal of Contemporary Medical Research 2017; 4(5):1208-1211.

21. Deborah Anne Burton, Keith Stokes, George M Hall et al., Physiological effects of exercise, Continuing Education in
Anaesthesia Critical Care \& Pain, Volume4,Issue-6,1December-2004,Pages:185-188, https://doi.org/10.1093/bjaceaccp/mkh050

22. MayureshJ Baheti et al., Ergonomics: A pain free dentistry for professionals. Department of Orthodontics Aug 2014.

23. Ashley $\mathrm{k}$ cole at el., National Athletic Trainers Association, Scapular Bracing and Alteration of Posture and Muscle Activity in Overhead Athletes With Poor Posture: Randomized Controlled Trial, 2013 JanFeb; 48(1): 12-24

24. Tania Pizzari et al., Modified a shrug exercise can facilitate the upward rotator muscles of scapula. Bristol, Avon. 2014 Feb.

25. Kamalanathan, P Banu H, Shabreen et al., International Journal of Pharmaceutical and Clinical Research; Effects of Upper Limb Strengthening exercises on handwriting speed in Undergraduate Student, 2017VL -9 DO- 10.25258\IJPCR.v9i6.8778.

How to cite this article: Simulia Dhinju B, Paulraj M, Manjula Devi K. Impact of pinch strength training along with postural education on upper extremity functional performance among dentist. Int J Health Sci Res. 2021; 11(5): 241-247. DOI: https://doi.org/10.52403/ ijhsr.20210539 